Z. klin. Chem. u. klin. Biochem.

9. Jg., S. $348-355$, Juli 1971

\title{
Fehleranalyse der Messung von Serumenzymaktivitäten
}

\author{
Von A. Nötges und ANNeltes Englhardt \\ Aus der 2. Medizinischen Klinik (Direktor: Prof. Dr. K. Oberdisse) der Universität Düsseldorf, und der Medizinischen \\ Klinik (Direktor: Prof. Dr. G. A. Martini) der Universität Marburg/Labn
}

(Eingegangen am 3. Februar 1970)

In vorliegenden Untersuchungen wurden Fehlerquellen, die die Reproduzierbarkeit von Enzymmessungen beeinflussen, geprüft. Die Bedeutung einzelner Teilschritte des Meßvorgangs für die Reproduzierbarkeit der Messung wurde untersucht und statistisch berechnet. Fehlerquellen der Vorphase sind unvollständiger Ablauf unspezifischer Begleitreaktionen und unzureichende Einstellung der vorgeschriebenen Reaktionstemperatur. Eine Untersuchung des Vorlaufs für die Enzyme Aspartattransaminase, Alanintransaminase, $\alpha-\mathrm{Hy}-$ droxybutyratdehydrogenase zeigte, daß dieser für die beiden Transaminasen rascher beendet ist als für $\alpha$-Hydroxybutyratdehydrogenase. Der statistische Vergleich des methodischen Fehlers einzelner Teilschritte mit Hilfe des F-Tests zeigte die Utberlegenheit der automatischen Registrierung gegenüber der manuellen und visuellen Technik. Es konnte gezeigt werden, daß der Vorgang der Auswertung des kinetischen Ablaufs im Anschluß an die Registrierung den gesamten Fehler der Messung erheblich beeinflußt.

Ergebnisse der Qualitätskontrolle von Enzymen und anderen Laboratoriumsuntersuchungen aus dem eigenen Laboratorium werden angegeben und mit den Ergebnissen anderer Untersucher verglichen.

\section{The analysis of error in the measurement of serum enzyme activities}

In the present work, the sources of error that influence the reproducibility of enzyme measurement were tested. The significance of the individual steps : of the measuring procedure for the reproducibility of the measurement was investigated and calculated statistically.

Sources of error in the preliminary phase are the failure of unspecific side reactions to reach completion, and failure to achieve the required reaction temperature. The first enzymic reaction stage of the aspartic transaminase and alanin transaminase reaches completion faster than that of $\alpha$-hydroxybutyric dehydrogenase. The statistical comparison of the methodical error of the individual stages with the aid of the F-test demonstrated the superiority of automatic tecording over the manual and visual technique. It was shown that the process, for the evaluation of the kinetics of the reaction, linked to a recording system, considerably influences the total error of the measurement. Results of the quality control of enzyms and other investigations from this laboratory are reported and compared with the results of other authors.

Von vielen Untersuchern wird auf die relativ hohe Streuung von Enzymaktivitätsmessungen aufmerksam gemacht. Es wird vielfach diskutiert, daß zu den speziellen Fehlerquellen, die bei anderen klinischchemischen Untersuchungen vorkommen können, noch weitere Irrtümer kommen, die sich aus den besonderen Eigenschaften der Enzyme selbst ergeben. Zu diesen gehören Temperatur- und $\mathrm{pH}-$ Empfindlichkeit, $\mathrm{Ab}$ hängigkeit von der Substratkonzentration, Empfindlichkeit gegen Störfaktoren in kleinsten Konzentrationen. Darüberhinaus umfaßt die Enzymanalyse außer den üblichen Analysenschritten (Reaktionsansatz, Photometrie, Auswertung) weitere spezifische Teilschritte, die ebenfalls Ursache von Fehlern sein können.

Nach Bürtner (1) reichen die Schritte einer Analyse, die bei der Prüfung der Zuverlässigkeit einer Methode beachtet werden müssen, von der Probennahme bis zur Beurteilung. Feblerquellen der Probenentnahme, die besonders bei Enzymmessungen beachtet werden müssen, wurden vielfach untersucht. Diese sind vor allem Hämolyse und Mikrohämolyse (2), Thrombocytenzerfall bei der Gerinnung $(3,4)$ und Alterung der Seren (5). Fehlerquellen, die die Reproduzierbatkeit von Enzymmessungen während des eigentlichen Meßvorgangs beeinflussen, sind bisher nicht im einzelnen geprüft worden.

In eigenen Untersuchungen wurde eine Analyse des Meßvorgangs selbst vom Testansatz bis zur Auswertung durchgeführt. Die Bedeutung der einzelnen Teilschritte für die Reproduzierbarkeit der Messung wurde geprüft. Die Teilschritte der Enzymmessungen, die Fehlermöglichkeiten enthalten, sind in Abbildung 1 zusammengestellt. Die eigenen Untersuchungen erfassen lediglich die Fehlerquellen des Analysenvorgangs.

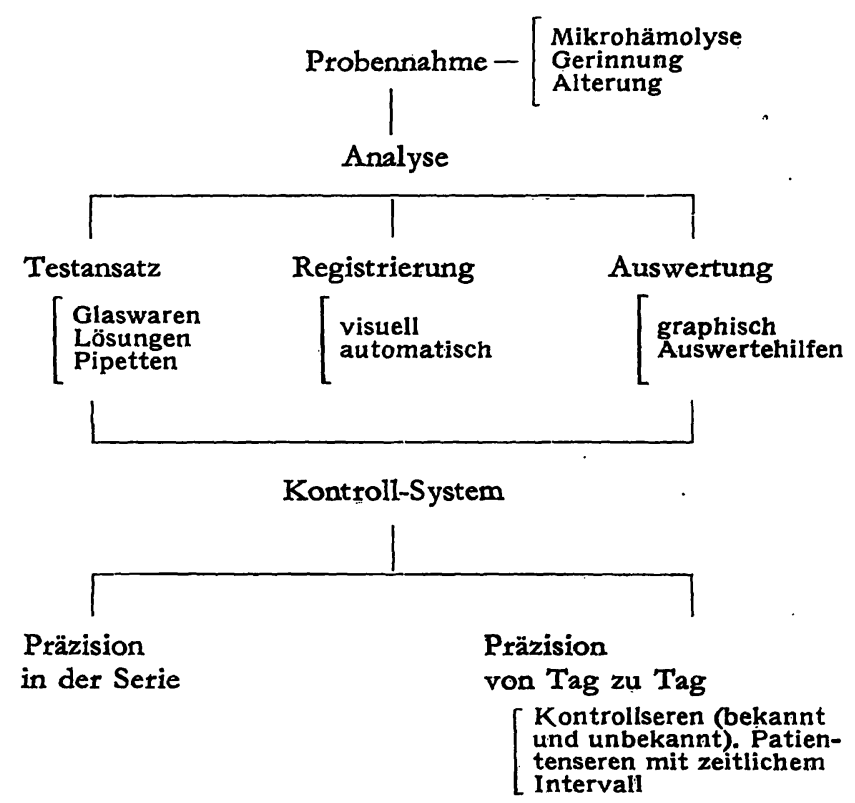

Abb. 1 Einzelschritte und Kontrollmöglichkeiten von Enzymaktivitäts- 


\section{Methodik}

\section{Messung der Enzymaktivitäten}

1. Alanintransaminase (L-Alanin: 2-Oxoglutarat-aminotransfcrase (EC 2.6.1.2)).

Reagenzien: L-Alanin 98proz., p. a.; NADH, di-Natrium-Salz, Gehalt enzymatisch 78\%, Lactatdehydrogenase aus Kaninchenmuskel, (LDH), Kristallsuspension in 2,2M Ammoniumsulfatlösung pH etwa 7,0, spez. Aktivität etwa $360 \mathrm{u} / \mathrm{mg}$; 2-Oxoglutarat. Reaktionsgemisch: 30 Teile L-Alanin, $80 \mathrm{~mm}$ in Phosphatpuffer $100 \mathrm{~mm}, \mathrm{pH} 7,4 ; 0,5$ Teile NADH $12 \mathrm{~mm}$; 0,5 Teile LDH-Suspension.

Test: Reaktionsgemisch $500 \mu \mathrm{l}$, Serum $100 \mu \mathrm{l}$. Nach 5 Min. Vorinkubation bei $25^{\circ}$ Start mit 2-Oxoglutarat $20 \mu 1250 \mathrm{~mm}$. Temperatur $25^{\circ}$. Wellenlänge $334 \mathrm{~nm}$. Halbmikroküvette $d=1 \mathrm{~cm}$

2. Aspartattransaminase (L-Aspartat: 2-Oxoglutarat-aminotransferase (EC 2.6.1.1)).

Reagenzien: L-Aspartat, Gehalt enzymatisch 98\%; NADH siebe oben; Malatdehydrogenase $(\mathrm{MDH})$ aus Schweineherz, Suspension in 3,2M Ammoniumsulfatlösung, $\mathrm{pH}$ etwa 6,0 , spez. Aktivität $1100 \mathrm{u} / \mathrm{mg}$; 2-Oxoglutarat.

Reaktionsgemisch: 30 Teile L-Aspartat $40 \mathrm{~mm}$ in Phosphatpuffer $100 \mathrm{~mm}, \mathrm{pH} 7,4 ; 0,5$ Teile NADH $12 \mathrm{~mm} ; 0,5$ Teile MDH-Suspension.

Test: Reaktionsgemisch $500 \mu \mathrm{l}$. Serum $100 \mu \mathrm{l}$. Nach 5 Min. Vorinkubation bei $25^{\circ}$ Start mit 2-Oxoglutarat $20 \mu 1250 \mathrm{~mm}$. Temperatur $25^{\circ}$. Wellenlänge $334 \mathrm{~nm}$. Halbmikroküvette $\mathrm{d}=1 \mathrm{~cm}$.

3. Lactatdehydrogenase (L-Lactat: NAD oxydoreduktase (EC 1.1.1.27)).

Reagenzien: Brenztraubensäure, krist. Mono-Natriumsalz, Gehalt enzymatisch $78 \%$; NADH wie oben.

Reaktionsgemisch: 1 Teil NADH $14 \mathrm{~mm} ; 100$ Teile NatriumPyruvat $0,15 \mathrm{~mm}$ in Phosphatpuffer $50 \mathrm{~mm}, \mathrm{pH} 7,5$.

Test: Reaktionsgemisch $1000 \mu \mathrm{l}$, Serum $100 \mu \mathrm{l}$. Temperatur 25, Wellenlänge $334 \mathrm{~nm}$. Halbmikroküvette $\mathrm{d}=1 \mathrm{~cm}$.

4. $\alpha$-Hydroxybutyratdehydrogenase, Lactatdehydrogenasen-Isoenzym (2-Hydroxybutyrat: NAD Oxydoreduktase (EC 1.1.1.30)). Reagenzien: 2-Oxobuttersäure p. a.; $\mathrm{NADH}$ wie oben.

Reaktionsgemisch: 60 Teile Natrium-2-Oxobutyrat $3 \mathrm{~mm}$ in Phosphatpuffer $50 \mathrm{~mm}$; pH 7,5; 1 Teil NADH $12 \mathrm{~mm}$.

Test: Reaktionsgemisch $1000 \mu \mathrm{l}$, Serum $50 \mu \mathrm{l}$. Temperatur $25^{\circ}$, Wellenlänge $334 \mathrm{~nm}$. Halbmikroküvette $d=1 \mathrm{~cm}$.

5. Alkalische Phosphatase (Orthophosphorsäuremonoester phosphohydrolase (EC 3.1.3.1)).

Test: $p$-Nitrophenylphosphat $5,5 \mathrm{~mm}$ und $0,5 \mathrm{~mm} \mathrm{MgCl}_{2}$ in Glycinpuffer $50 \mathrm{mM}, \mathrm{pH} 10,5,500 \mu \mathrm{l}$; Serum $50 \mu \mathrm{l}$, Temperatur $25^{\circ}$. Wellenlänge $405 \mathrm{~nm}$, Halbmikroküvette $d=1 \mathrm{~cm}$.

6. Leucinaminopeptidase (EC 3.4.1.1).

Test: Leucin-p-nitroanilid $10 \mathrm{~mm}$ in Phosphatpuffer $50 \mathrm{~mm}$, pH 7,2,500 $\mu \mathrm{l}$; Serum $50 \mu \mathrm{l}$. Temperatur $25^{\circ}$. Wellenlänge $405 \mathrm{~nm}$. Halbmikroküvette.

Für alle Messungen wurden Mikroliterpipetten des MarburgSystems verwendet. Die Messungen erfolgten am Photometer Eppendorf mit Kompensationsschreiber und thermokonstanter Küvettenwechselautomatik. Vorschubgeschwindigkeit $2 \mathrm{~cm} / \mathrm{Min}$. Registrierlänge $0,5 \mathrm{~cm}$. Lediglich bei der Messung der Leucinaminopeptidase war die Vorschubgeschwindigkeit $0,5 \mathrm{~cm} / \mathrm{Min}$. Variationen dieser Meßanordnung siehe unter $2 \mathrm{~b}$.

Auswerlung:

Die Auswertung exfolgte

a) mit dem Enzymtestauswerter Eppendorf. Ablesung durch Schätzung auf etwa $0,1^{\circ}$.

b) graphisch durch Verbinden der Meßstrecken zu einer Graden, Ablesen der Extinktion an der Ordinate und Berechnung der Enzymeinheiten. Bei dieser Auswertung erfolgte die Ermittlung der Extinktionsänderung/Min. entweder bei einer konstanten
Registrierlänge von $10 \mathrm{~cm}$ oder durch Verlängerung der Geraden bis zu einem Schnittpunkt mit dem Registriernetz.

c) mit Winkelmesser und Berechnung von $\operatorname{tg} \alpha$.

II. Methoden zur Prüfung der Fehlerquellen

Messung der Enzymaktivitäten

a) Vorphase

Für die beiden Transaminasen und $\alpha$-Hydroxybutyratdehydrogenase wurde der Verlauf während der Vorinkubation gemessen. Der Reaktionsansatz ohne Substrat wurde vor dem Start bei konstanter Temperatur von $25^{\circ}$ unter fortwährender automatischer Registrierung so lange inkubiert, bis die Extinktion über $5 \mathrm{Min}$. konstant blieb, dann wurde die Reaktion durch Zusatz von Substrat gestartet.

b) Testansatz

Der Testansatz wurde in zweierlei Weise für die beiden Transaminasen, Lactatdehydrogenase und $\alpha$-Hydroxybutyratdehydrogenase durchgeführt:

1. Testansatz aus Reaktionsgemisch und Serum (siehe Tcil 1).

2. Testansatz mit Einpipetticren der Lösungen von Substraten, Hilfsenzymen und Coenzymen jewreils als definiertes Volumen.

Methodik für Testansatz mit Einzelpipetticrung (II, b, 2). Alanintransaminase:

L-Alanin $80 \mathrm{~mm}$ in Phosphatpuffer $100 \mathrm{~mm}$, pH 7,4, $600 \mu \mathrm{l}^{1}$ ); $\mathrm{NADH} 12 \mathrm{~mm} 10 \mu \mathrm{l}$; Lactatdehydrogenase-Suspension $10 \mu \mathrm{l}$; Serum $10 \mu \mathrm{l}$. Nach Vorinkubation (5 Min. $2^{\circ}$ ) Start mit 2-Oxoglutarat $250 \mathrm{~mm} 20 \mu \mathrm{l}$.

Aspartattransaminase:

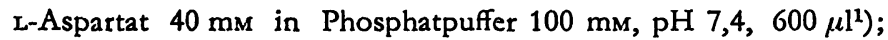
$\mathrm{NADH} 12 \mathrm{~ms} 10 \mu \mathrm{l}$; Malatdchydrogenase-Suspension $10 \mu \mathrm{l}$; Serum $100 \mu \mathrm{l}$. Nach Vorinkubation (5 Min. 25 ) Start mit 2Oxoglutarat $250 \mathrm{~mm} 20 \mu l$.

Lactatdehydrogenase:

Natrium-Pyruvat $0,15 \mathrm{~mm}$ in Phosphatpuffer $50 \mathrm{~mm}, \mathrm{pH} 7,5$, $100 \mu \mathrm{l}$; NADH $14 \mathrm{~mm} 10 \mu \mathrm{l}$, Serum $100 \mu \mathrm{l}$.

$\alpha$-Hydroxybutyratdehydrogenase:

Natrium-2-Oxobutyrat $3 \mathrm{~mm}$ in Phosphatpuffer $50 \mathrm{~mm}, \mathrm{pH} 7,5$, $600 \mu \mathrm{l}$; NADH $12 \mathrm{~mm} 10 \mu \mathrm{l}$; Serum $20 \mu \mathrm{l}$. Übrige Enzymaktivitätsmessungen siehe unter $I$.

c) Meßvorgang

Der Ablauf der kinetischen Reaktion wurde auf zweierlei Weise verfolgt:

1. Durch visuelle Ablesung am Photometer Eppendorf mit thermokonstantem Küvettenhalter.

Es wurde in Abstand von $1 \mathrm{Min}$. im ganzen 4 Min. lang gemessen, wobei die Küvetten von Hand alle 20 Sek. in den Strahlengang gebracht. wurden.

2. Durch Registrieren mit dem Kompensationsschreiber zum Photometer Eppendorf.

Es wurden folgende Papiervorschubgeschwindigkeiten und Registrierlängen verwendet:

Vorschubgeschwindigkeit $1 \mathrm{~cm}$ bzw. $0,5 \mathrm{~cm} / \mathrm{Min} .$, Registrierlänge $0,1 \mathrm{~cm}$.

Vorschubgeschwindigkeit $1 \mathrm{~cm}$ bzw. $2 \mathrm{~cm} /$ Min., Registrierlänge $0,5 \mathrm{~cm}$.

\section{Präzisionskontrolle}

Die Präzision in der Serie wurde für alle Enzyme durch 16 fache Bestimmung in einem Ansatz ermittelt. Die Präzision von Tag zu Tag wurde im Routinclaboratorium der Medizinischen Universitätsklinik Düsseldorf unter Standardbedingungen in folgender Weise gepriift:

1) Das Volumen von $600 \mu \mathrm{l}$ wurde mit einer Spritzenpipette (Ultrasept) pipetticrt, alle übrigen Volumina mit Mikroliterpipetten des Marburg-Systems. 
Tägliche Kontrollen wurden mit Kontrollseren (Enza-trol) durchgeführt und die Werte auf Kontrollkarten übertragen. Für alle übrigen klinisch-chemischen Analysen wurden ebenfalls Qualitätskontrollen mit Lab-trol oder Moni-trol durchgeführt. Für die statistische Auswertung wurde eine Kontrollperiode von über 3 Monaten, vom Beginn des jetzigen Kalenderjahres an, herangezogen, in der eine einzige Charge des Kontrollserums verwendet wurde. Das Laborpersonal wurde nicht darüber informiert, da $\beta$ das Zahlenmaterial zur Veröffentlichung ausgewertet werden sollte.

Für die Kontrolle von Enzymmessungen von Tag zu Tag wurde eine zweite Kontrollperiode ausgewertet (Laboratorium der Univerisät Marburg). In dieser wurden anstelle von im Handel erhältlichen Seren Patientenseren mit einer in einem definierten Bereich liegenden Aktivität, die aus der Serie eines Tages entnommen worden, am darauffolgenden Tag (etwa 24 Stdn. später) nochmals gemessen. Diese Kontrollen wurden durchgeführt für folgende Enzyme: Aspartattransaminase, Alanintransaminase, Lactatdehydrogenase und alkalische Phosphatase. Die Berechnung von $\overline{\mathrm{x}}$ und $\mathrm{s}$ erfolgte aus den Doppelbestimmungen an hintereinanderliegenden Tagen.

Diese Methode der Qualitätskontrolle wurde zunächst von KREUTZ im Klinisch-Chemischen Laboratorium der Universität Marburg/Lahn eingeführt und wird bis heute dort routinemäßig durchgeführt.

\section{Ergebnisse}

Die Vorphase

Im Reaktionsansatz spielen sich bereits vor Zusatz des Substrats Umsetzungen $a b$, da die serumeigenen Enzyme mit ihren ebentalls im Serum vorkommenden Substraten in Reaktion treten. Daher muß vor der Messung Reaktionsstillstand abgewartet werden. Der Ablauf dieser Vorphase wurde für die drei Enzyme Aspartattransaminase, Alanintransaminase und $\alpha$-Hydroxybutyratdehydrogenase geprüft, da bei diesen der Start mit Substrat durchgeführt werden muß. Dagegen können die Messungen von Leucinaminopeptidase, alkalischer Phosphatase und Lactatdehydrogenase mit Serum gestartet werden. Die Substrate der beiden ersten Enzyme sind im Serum nicht nachweisbar. Für Lactatdehydrogenase erfolgt keine Störung durch serumeigenes Pyruvat, da dieses im Überschuß zugesetzt wird. Für die beiden Transaminasen war der Vorlauf bereits nach $2 \mathrm{Min}$. beendet, für $\alpha$-Hydroxybutyratdehydrogenase aber erst nach $8 \mathrm{Min}$. Das bedeutet, daß für $\alpha$-Hydroxybutyratdehydrogenase eine Wartezeit von $10 \mathrm{Min}$. vor dem Start zu empfehlen ist, für die beiden Transaminasen dagegen nur von $5 \mathrm{Min}$.

\section{Fehleranalyse der einzelnen Teilschritte des Meßvorgangs}

Priifung mit dem F-Test.

Der Meßvorgang setzt sich zusammen aus den Teilschritten Pipettierung, Registrierung und Auswertung. Tabelle 1 zeigt den statistischen Vergleich der Bedeutung der einzelnen Teilschritte des Analysenvorgangs für den Gesamtfehler. Für jeden dieser Teilschritte. wurden eine bis mehrere methodische Variationen angewendet. Die Streuungen der Einzelwerte, die auf diese Weise erhalten wurden, wurden mit Hilfe des F-Tests verglichen. Zunächst wurde eine Serie mit einzelnen Pipettierschritten und eine weitere mit einer Testmischung verglichen. Durch Reduzierung der Pipettierschritte bei der Herstellung des Testansatzes wurde eine signifikante Verbesserung der Präzision sowohl bei manuellem Küvettenwechsel als auch in der Wechselautomatik erzielt. In einer weiteren Serie wurde die automatische Registrierung mit der manuellen und visuellen Technik verglichen. Bei letzterer erfolgt der Küvettenwechsel durch manuelle Bedienung des Schwenkhebels und nachfolgende yisuelle Registrierung der Extinktion in konstanten Intervallen. In der Vergleichsserie wurde eine signifikant bessere Präzision bei automatischer Registrierung gefunden.

In der nächsten Serie wurde automatisch registriett, die Technik der Auswertung wurde abér variiert. Die einfachste Form der Auswertung bei graphischer Darstellung ist die direkte Ablesung des $\triangle \mathrm{E}$ anhand der Steigung der Geraden an der zugehörigen Skala. Es zeigte sich, daß aie Streuung der Werte signifikant kleiner wird, wenn man nicht bei konstanter Meßperiode abliest, sondern die Registrierlinie bis zum Schnittpunkt der Geraden mit dem Netz des Registrierpapiers verlängert (Auswertung mit variabler $\mathrm{Meß}-$ periode). Im letzteren Fall werden die Extinktionsänderungen an der Skala auf 0,01 genau abgelesen,

Tab. 1

Analyse der Fehler des Meßvorgangs und der Auswertung von Enżymmessungen

Messung der Lactatdehydrogenase-Aktivität eines Patienten-Serums. Test im Mikroliter-System mit Marburg-Pipetten. Photometer Eppendorf mit Kompensationsschreiber und 6 Küvettenwechselautomatik

Temperatur $25^{\circ}$; Wellenlänge $334 \mathrm{~nm} . \mathrm{n}=18, \pm \mathrm{s}\left(\Delta \mathrm{E} / \mathrm{Min} . \times 10^{3}\right), \mathrm{C}=2,22 \mathrm{~F}=\frac{\mathrm{s}_{1}^{2}}{\mathrm{~s}_{2}^{2}}$

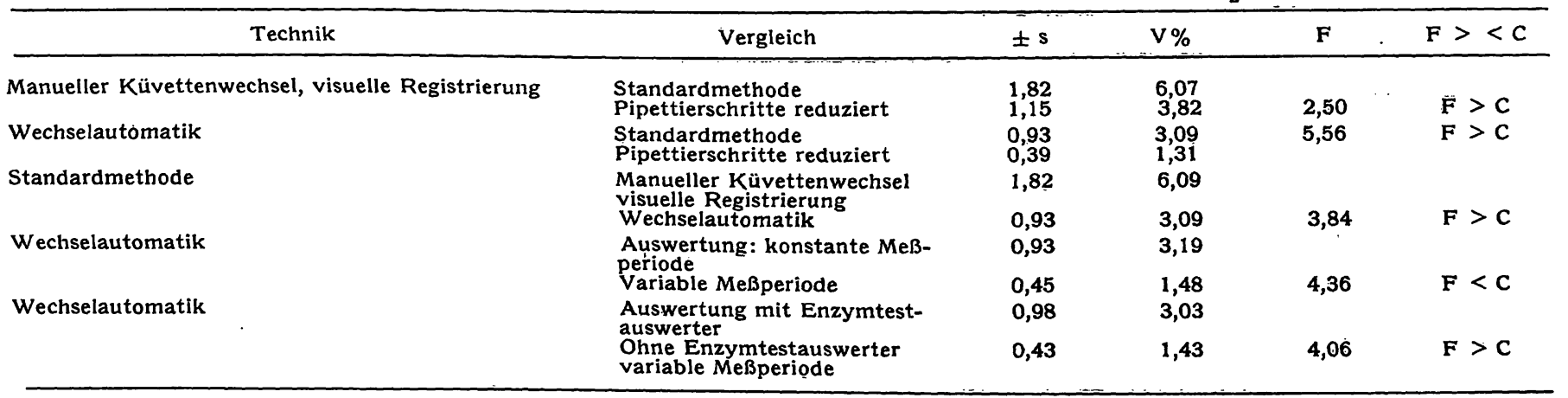


während im anderen Fall geschätzt werden muß, da die Gerade die Skala in der Regel zwischen den Markierungen schneidet. In einer weiteren Serie wurde der Enzymtestauswerter Eppendorf verwendet. Dieser ruißt die Steigung in Winkelgraden, die mit Hilfe des tg $\alpha$ in Enzymeinheiten umgewandelt wurden.

Die Streuung bei Anwendung dieses Hilfsmittels entsprach bei Schätzung der Dezimalstellen derjenigen der graphischen Auswertung mit konstantem Intervall und derjenigen Auswertung mit Winkelmesser und Berechnung des tg $\alpha$. Dagegen war die Präzision bei Auswertung mit variabler Meßperioce signifikant besser, wenn ohne Auswerthilfe abgelesen wurde (Abb. 2 und 3).

Unterschiedliche Papiervorschübe und Registrierlängen ergaben keine signifikante Änderung der Präzision. Am günstigsten erwies sich ein Papiervorschub von $2 \mathrm{~cm} /$ Min. und eine Registrierlänge von $0,5 \mathrm{~cm}$. Unter diesen Bedingungen machen sich kleine Schwankungen im Ablauf der Reaktion am wenigsten bemerkbar, die graphische Darstellung wird übersichtlicher.

\section{Varianzanalyse}

Durch Varianzanalyse wurde geprüft, ob die Gesamtstreuung des Tests durch die Auswertung deutlich beeinflußt wird. Geprüft wurde die Auswertung mit konstanter Meßperiode und die Ablesung am Enzymtestauswerter. In beiden Fällen fand sich kein signifikanter Unterschied der Streuung zwischen den Serien von der Streuung innerhalb der Serien. Das bedeutet, da $\beta$ durch die Auswertung ein Versuchsfehler entsteht, der die gesamte Streuung erheblich beeinflußt (Tab. 2).

\section{Qualitätskontrolle}

Die Untersuchungen haben gezeigt, daß einige Fehlerquellen der Enzymmessungen bei guter Meßtechnik vermieden werden können. Andere dagegen können nicht völlig ausgeschaltet werden, z. B. Verwendung von Hilfsmitteln bei der Auswertung. Es wurde nun geprüft, ob bei optimaler Meßtechnik die Werte der Enzymmessungen ebenso zuverlässig sind wie andere Untersuchungsmethoden.

\section{Qualitätskontrolle im eigenen Laboratorium}

Im Routinelaboratorium der Klinik wurde die Qualitätskontrolle aller klinisch-chemischer Methoden mit den Kontrollseren Lab-trol, Enza-trol und Moni-trol durchgeführt. Die Meßbedingungen des Laboratoriums entsprachen den vorher ermittclten Anforderungen an

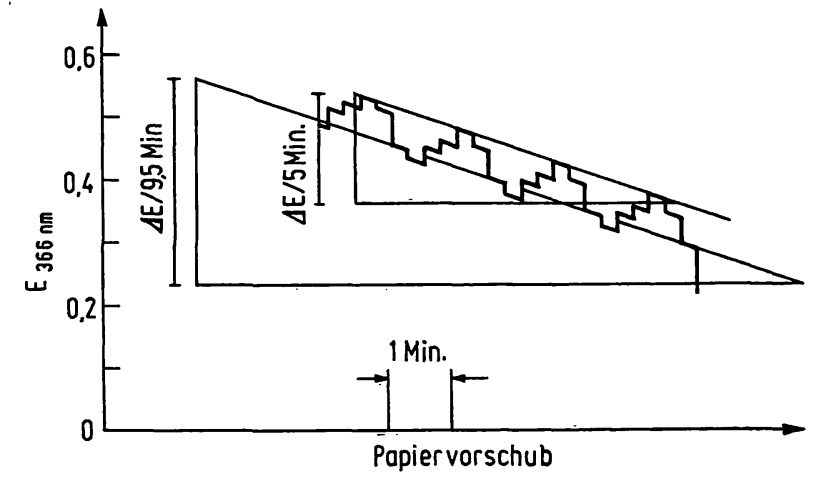

Abb. 2

Auswertung der Aktivitätsbestimmung der Lactatdehydrogenase mit konstanter und variabler Meßperiode. Photometer Eppendorf mit Kompensationsschreiber und 6-Küvettenwechselautomatik Papiervorschub $2 \mathrm{~cm} / \mathrm{Min}$., Registrierlänge $0,5 \mathrm{~cm}$
Abb. 3

Enzymtestauswerter (Netheler und Hintz, Eppendorf-Gerätebau) mit direkter Ablesung von Enzymaktivitäten

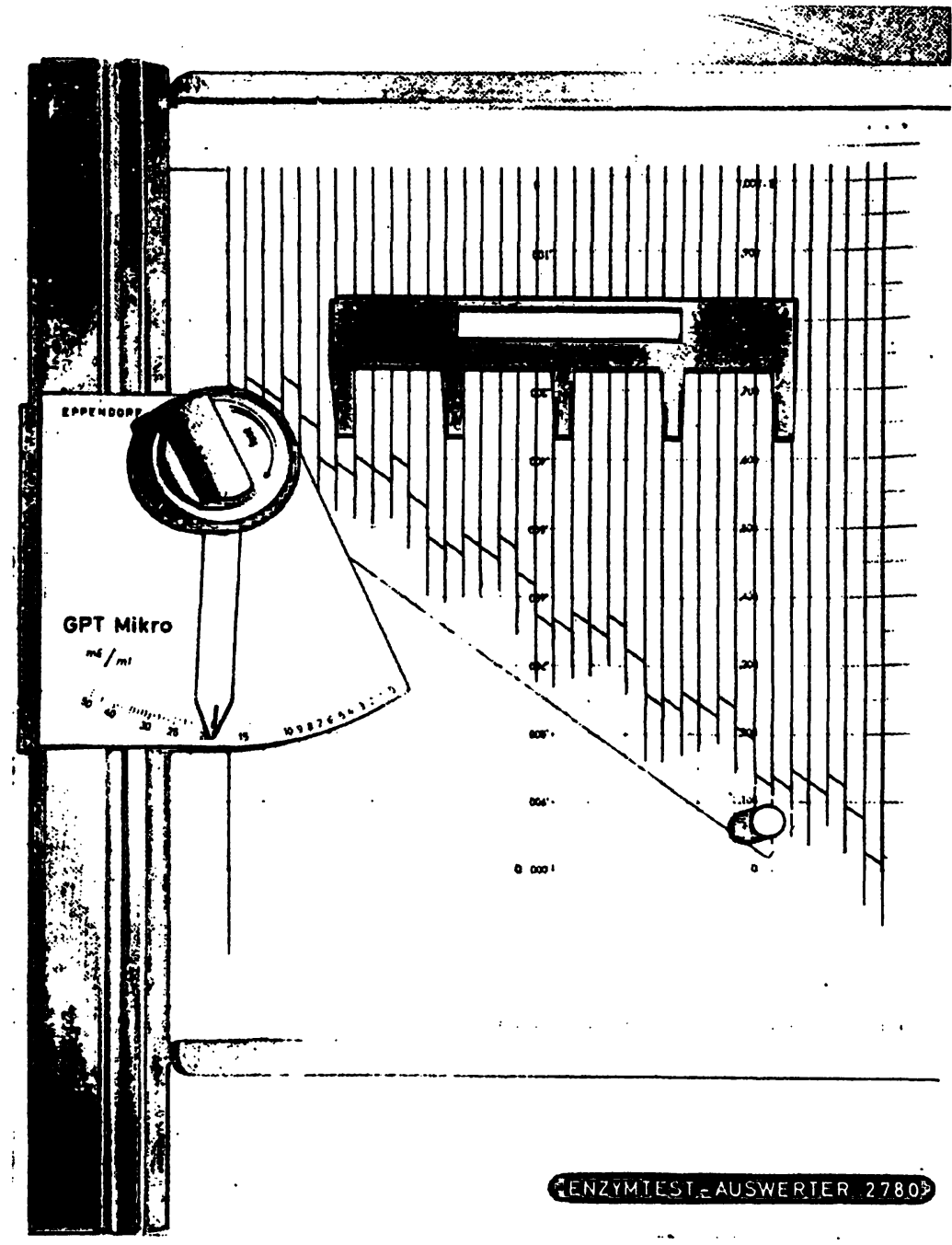


Tab. 2

Die Lactatdehydrogenase-Aktivität eines $M$ Iisch-Serums (10 gesunde Spender) wurde als 8 fach-Bestimmung gemessen. Die 8 Registrierungen wurden jeweils $8 \mathrm{mal}$ mit dem Enzymtestauswerter ausgewertet. Photometer Eppendorf mit Küvettenwechselautomatik und Kompensationsschreiber. Papiervorschub $2 \mathrm{~cm} /$ Min., Registrierlänge $0,5 \mathrm{~cm}$

Die zur Varianzanalyse benötigten Größen wurden nach folgendem Schema berechnet

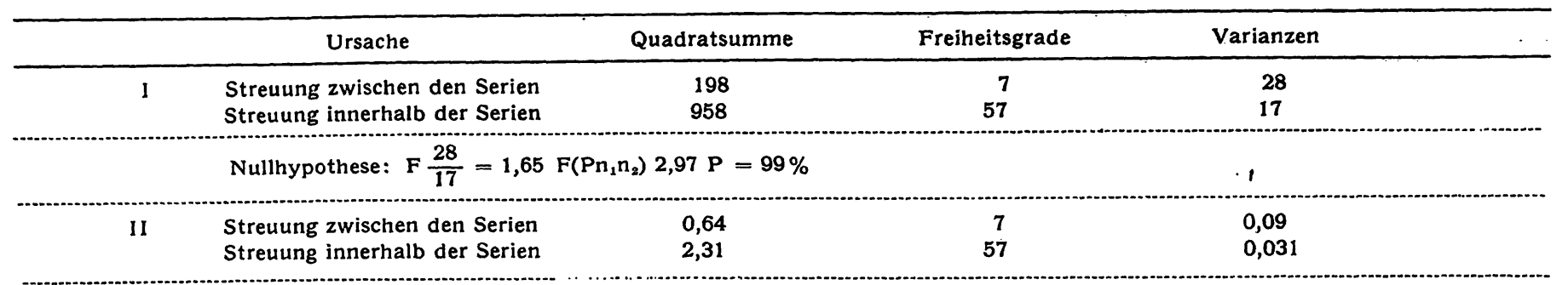

Nullhypothese: $F \frac{0,09}{0,031}=0,290 F\left(P n_{1} n_{2}\right) P=99 \%$ (s. o.)

I Die Werte wurden transformiert ( $\Delta \mathrm{E} / \mathrm{Min}$.).

II Die Werte wurden angegeben in Winkelgraden.

Tab. 3

Präzisionsvergleich der Analysen von Enzymmessungen und anderen Laboratoriumsmethoden bei Bestimmungen in der Serie und von Tag zu Tag. Kontrollseren Moni-trol I und Enza-trol. Kontrollperiode 91 Tage

\begin{tabular}{|c|c|c|c|c|c|c|}
\hline \multirow{2}{*}{ Analyse } & \multicolumn{3}{|c|}{ Präzision in der Serie } & \multicolumn{3}{|c|}{ Präzision von Tag zu Tag } \\
\hline & $\overline{\mathbf{x}}$ & $\mathbf{s}$ & $\mathrm{V} \%$ & $\overline{\mathbf{x}}$ & $\mathbf{s}$ & $\mathrm{V} \%$ \\
\hline $\begin{array}{l}\text { Bilirubin }(\mathrm{mg} / 100 \mathrm{ml}) \\
\text { Harnstoff }(\mathrm{mg} / 100 \mathrm{ml}) \\
\text { Harnsäure }(\mathrm{mg} / 100 \mathrm{ml}) \\
\text { Anorg. Phosphat }(\mathrm{mg} / 100 \mathrm{ml}) \\
\mathrm{Ca}^{++}(\mathrm{mVal} / \mathrm{l}) \\
\mathrm{K}^{+}(\mathrm{mVal}(\mathrm{l}) \\
\mathrm{Na}^{+}(\mathrm{mVal} / \mathrm{l}) \\
\mathrm{Cl}-(\mathrm{mVal} / \mathrm{l})\end{array}$ & $\begin{array}{r}3,68 \\
29,10 \\
4,30 \\
3,41 \\
5,02 \\
4,15 \\
138,50 \\
103,00\end{array}$ & $\begin{array}{l}0,06 \\
0,48 \\
0,06 \\
0,07 \\
0,08 \\
0,08 \\
0,92 \\
0,62\end{array}$ & $\begin{array}{l}1,62 \\
1,71 \\
1,48 \\
2,08 \\
1,62 \\
1,98 \\
0,67 \\
0,61\end{array}$ & $\begin{array}{r}3,44 \\
43,80 \\
4,30 \\
3,88 \\
5,00 \\
4,20 \\
139,00 \\
104,00\end{array}$ & $\begin{array}{l}0,09 \\
1,26 \\
0,23 \\
0,15 \\
0,20 \\
0,14 \\
3,01 \\
0,92\end{array}$ & $\begin{array}{l}2,51 \\
2,87 \\
5,24 \\
3,86 \\
4,04 \\
3,26 \\
2,17 \\
0,89\end{array}$ \\
\hline $\begin{array}{l}\text { Alanin-Transaminase }(u / l) \\
\text { Aspartat-Transaminase (ul/) } \\
\text { Alk. Phosphatase }(u / 1) \\
\text { Saure Phosphatase }(u / 1) \\
\text { Lactatdehydrogenase }(u / l)\end{array}$ & $\begin{array}{r}49,70 \\
49,40 \\
54,00 \\
22,60 \\
194,00\end{array}$ & $\begin{array}{l}1,72 \\
2,01 \\
2,00 \\
0,87 \\
7,09\end{array}$ & $\begin{array}{l}3,46 \\
4,07 \\
3,70 \\
3,85 \\
3,65\end{array}$ & $\begin{array}{r}43,00 \\
48,00 \\
171,00 \\
23,00 \\
189,00\end{array}$ & $\begin{array}{r}3,63 \\
3,83 \\
18,90 \\
2,20 \\
17,40\end{array}$ & $\begin{array}{r}8,44 \\
7,97 \\
11,09 \\
9,56 \\
-\quad 8,76\end{array}$ \\
\hline
\end{tabular}

apparativer Ausrüstung und Meßtechnik. Bei der Auswertung wurde der Enzymtestauswerter Eppendorf benutzt, auf den aus Gründen der Rationalität nicht verzichtet werden konnte. Vielfachbestimmungen in der Serie und Qualitätskontrolle wurden jeweils von derselben Assistentin durchgeführt. Tabelle 3 zeigt Mittelwerte, Standardabweichungen und Variationskoeffizienten von Enzymmessungen und analytischen Methoden.

Die Werte für s wurden für jedes einzelne Enzym mit den Standardabweichungen der klinisch-chemischen Methoden verglichen. Es zeigte sich, daß vor allem bei Kontrollen von Tag zu Tag die Summe der Fehler von Enzymmessungen bis auf wenige Ausnahmen signifikant größer war als die der übrigen Methoden (Tab. $4 a$ und $4 b)$.

\section{Vergleich mit den Ergebnissen anderer Laboratorien}

Die von anderen Autoren veröffentlichten Ergebnisse der Qualitätskontrolle für Enzyme zeigen ebenfalls relativ hohe Streuungen (Tab. 5). BürTNER hat vor allem auf diesen Befund hingewiesen (1). FREI betichtet über Variationen von Tag zu Tag (13), die im Normbereich wesentlich höher lagen als im pathologischen Bereich. Eine Erklärung hierfür sieht der Autor in der ungenügenden Empfindlichkeit der Ênzymmessungen im normalen Bereich.

\section{Febler, die die Präzision von Enzymmessungen beeinflussen}

Die Präzision von Enzymmessungen wird noch durch einen weiteren Faktor beeinflußt, der vom eigentlichen Meßvorgang unabhängig ist. Kontrollseren für Enzymmessungen unterscheiden sich von Patientenseren dadurch, daß sie entweder tiefgefroren oder lyophilisiert werden müssen. Einige Ergebnisse sprechen dafür, daß die Enzymaktivitäten in diesen Seren durch die Vorgänge des Lyophilisierens und Lösens bzw. Einfrierens und Tauens auch bei schonender Behandlung beeinflußt werden. KREUTZ (18) fand die höchsten Variationskoeffizienten bei Verwendung eines eingefrorenen Pool-Serums, das in Einzelportionen abgefüllt worden war und jeweils am Tag der Untersuchung aufgetaut wurde. Die Ergebnisse mit einem kommerziellen Kontrollserum (Enza-trol) entsprechen denen der übrigen Autoren und eigenen Ergebnissen. Dagegen waren die Variationskoeffizienten deutlich niedriger, wenn zur Qualitätskontrolle Patientenseren verwendet wurden, die an zwei hintereinanderfolgenden Tagen als Doppelbestimmungen gemessen wurden ${ }^{2}$ ). Voraussetzung war allerdings, daß die Aktivitäten dieser Kontrollseren nicht im normalen oder extrem hohen Bereich lagen (Tab. 6).

2) Die Kontrollen wurden von Herrn Doz. Dr. KREUTz im Laboratorium der Medizinischen Klinik der Universität Marburg durchgeführt. 
Tab. $4 a$ und $4 b$

Statistischer Vergleich der Varianzen von Enzymbestimmungen und anderer klinisch-chemischer Methoden, Prüfung der Hypothese $s_{1}^{2}=s_{2}^{2}$ gegen $s_{1}^{2}>s_{2}^{2} ; F=\frac{s_{1}^{2}}{s_{2}^{2}} \cdot C$ für Spalte A: 5,05 [Signifikanzzahl für $C\left(P, n_{1} n_{2}\right)(f u ̈ r ~ P=95 \%)$.]

Tab. $4 a$

\begin{tabular}{|c|c|c|c|c|c|c|}
\hline \multicolumn{7}{|c|}{$A$ in der Serie } \\
\hline Analysen & & $\begin{array}{c}\text { Aspartat- } \\
\text { transaminase }\end{array}$ & $\underset{\text { transaminase }}{\text { Alanin- }}$ & $\begin{array}{c}\text { Alkal. } \\
\text { Phosphatase }\end{array}$ & $\begin{array}{c}\text { Saure } \\
\text { Phosphatase }\end{array}$ & $\begin{array}{c}\text { Lactatdehy- } \\
\text { drogenase }\end{array}$ \\
\hline Bilirubin & $\mathbf{F}$ & $F^{6,31}>C$ & $F^{4,28}<C$ & $F^{5,25}>C$ & $F^{5,62}>C$ & $F^{5,07}>C$ \\
\hline Harnstoff & $\mathbf{F}$ & $F^{5,67}>C$ & $F^{4,14}<$ & $F^{4,69}<C$ & $F^{5,09}>C$ & $F^{4,58}>C$ \\
\hline Harnsäure & $\mathbf{F}$ & $F^{7,45}>C$ & $F^{5,37} \mathrm{C}$ & $F^{6,16} c$ & $F^{6,71}>C$ & $F^{6,02}>C$ \\
\hline Anorg. Phosphor & $\mathbf{F}$ & $F^{3,88}<C$ & $\mathrm{~F}^{2,79}<\mathrm{C}$ & $F^{3,20}<C$ & $\mathrm{~F}^{3,43}<\mathrm{C}$ & $\mathrm{F}^{3,08}<\mathrm{C}$ \\
\hline $\mathrm{Ca}++$ & $\mathbf{F}$ & $F \stackrel{6,25}{>} C$ & $F^{4,49}<C$ & $F^{5,10}>C$ & $F^{5,58}>C$ & $\mathrm{~F}^{9,00}<\mathrm{C}$ \\
\hline $\mathrm{K}^{+}$ & $\mathbf{F}$ & $F^{4,23}<C$ & $F^{3,05}<C$ & $F^{3,51}<C$ & $F^{3,83}<C$ & $F^{3,43}<C$ \\
\hline $\mathrm{Na}^{+}$ & $\mathbf{F}$ & $\stackrel{39,20}{\mathrm{~F}}>\mathrm{C}$ & $\stackrel{26,90}{\mathrm{~F}}>\mathrm{C}$ & $\stackrel{31,00}{F} \mathrm{C}$ & $\stackrel{30,35}{\mathrm{~F}}>\mathrm{C}$ & $\stackrel{30,28}{F}>C$ \\
\hline $\mathrm{Cl}^{-}$ & $\mathbf{F}$ & $\begin{array}{c}44,00 \\
F\end{array}$ & $\stackrel{32,17}{F}>C$ & $\stackrel{37,38}{F}=$ & $\stackrel{39,93}{F}>C$ & $\stackrel{35,40}{F}>C$ \\
\hline
\end{tabular}

Tab. $4 \mathrm{~b}$

\begin{tabular}{|c|c|c|c|c|c|c|}
\hline \multicolumn{7}{|c|}{ B von Tag zu Tag } \\
\hline Analysen & & $\begin{array}{c}\text { Aspartat- } \\
\text { transaminase }\end{array}$ & $\underset{\text { transaminase }}{\text { Alanin- }}$ & $\begin{array}{l}\text { Alkal. } \\
\text { Phosphatase }\end{array}$ & $\begin{array}{c}\text { Saure } \\
\text { Phosphatase }\end{array}$ & $\begin{array}{c}\text { Lactatdehy- } \\
\text { drogenase }\end{array}$ \\
\hline Bilirubin & $\begin{array}{l}\mathbf{F} \\
\mathbf{C}\end{array}$ & $\begin{array}{r}9,23 \\
1,98 \\
F>C\end{array}$ & $\begin{array}{c}10,29 \\
1,98 \\
F \stackrel{C}{ }\end{array}$ & $\begin{array}{r}17,96 \\
2,25 \\
F^{>}>C\end{array}$ & $\begin{array}{c}13,44 \\
1,98 \\
F>C\end{array}$ & $\begin{array}{r}2,95 \\
2,25 \\
F^{>} C\end{array}$ \\
\hline Harnstoff & $\begin{array}{l}\mathbf{F} \\
\mathbf{C}\end{array}$ & $\begin{array}{r}7,81 \\
1,77 \\
>\end{array}$ & $\begin{array}{r}8,72 \\
1,77 \\
F>C\end{array}$ & $\begin{array}{r}14,82 \\
2,05 \\
F^{>}>C\end{array}$ & $\begin{array}{c}11,11 \\
1,77 \\
>\end{array}$ & $F^{2,43}>C$ \\
\hline Harnsäure & $\stackrel{F}{\mathbf{C}}$ & $\begin{array}{r}2,23 \\
1,77 \\
F^{\prime}>C\end{array}$ & $\begin{array}{c}2,51 \\
1,77 \\
F^{>} C\end{array}$ & $\begin{array}{r}4,30 \\
2,05 \\
F^{\prime}>C\end{array}$ & $\begin{array}{c}3,25 \\
1,77 \\
F^{>} C\end{array}$ & $\begin{array}{c}<1 \\
2,05 \\
F\end{array}$ \\
\hline Anorg. Phosphor & $\stackrel{F}{\mathbf{C}}$ & $\mathrm{F}^{2,09} \begin{array}{c}1,69 \\
<\mathrm{C}\end{array}$ & $\mathrm{F}^{1,92} \begin{array}{c}2,01 \\
<\mathrm{C}\end{array}$ & $\begin{array}{c}3,22 \\
F^{2,27}>C\end{array}$ & $\begin{array}{c}2,40 \\
2,01 \\
F^{>} C\end{array}$ & $\begin{array}{r}<1 \\
2,05 \\
F^{<}\end{array}$ \\
\hline $\mathrm{Ca}^{++}$ & $\begin{array}{l}\mathbf{F} \\
\mathbf{C}\end{array}$ & $\begin{array}{r}3,90 \\
F^{1,99}>C\end{array}$ & $\begin{array}{r}4,40 \\
1,99 \\
F>C\end{array}$ & $\begin{array}{c}7,48 \\
2,19 \\
F^{>}>C\end{array}$ & $\begin{array}{r}5,59 \\
1,99 \\
F^{>}>C\end{array}$ & $\mathrm{~F}^{2,19}<\mathrm{C}$ \\
\hline $\mathrm{K}^{+}$ & $\underset{\mathbf{C}}{\mathbf{F}}$ & $\begin{array}{c}3,52 \\
1,91 \\
F^{>} C\end{array}$ & $\begin{array}{r}3,98 \\
1,91 \\
F^{>}>C\end{array}$ & $\begin{array}{r}6,72 \\
2,17 \\
F^{\prime}>C\end{array}$ & $\begin{array}{r}5,04 \\
1,91 \\
F^{\prime}>C\end{array}$ & $F^{2,17}<C$ \\
\hline $\mathrm{Na}^{+}$ & $\underset{C}{\mathbf{F}}$ & $\begin{array}{r}13,30 \\
1,95 \\
F>C\end{array}$ & $\begin{array}{r}15,14 \\
1,95 \\
F>C\end{array}$ & $\begin{array}{r}26,09 \\
2,22 \\
F^{>}>C\end{array}$ & $\begin{array}{r}18,62 \\
1,95 \\
F>C\end{array}$ & $\begin{array}{r}4,32 \\
2,22 \\
F^{>} C\end{array}$ \\
\hline $\mathrm{Cl}^{-}$ & $\mathbf{F}$ & $\begin{array}{c}81,49 \\
2,05 \\
F>C\end{array}$ & $\begin{array}{c}91,90 \\
2,05 \\
F^{>}>C\end{array}$ & $\begin{array}{r}156,70 \\
2,33 \\
F^{>}>C\end{array}$ & $\begin{array}{c}121,00 \\
2,05 \\
F^{>}>C\end{array}$ & $\begin{array}{c}25,74 \\
2,33 \\
F^{>} C\end{array}$ \\
\hline
\end{tabular}

Tab. 5

Qualitätskontrolle der Enzyme: + Pool-Serum, ++ Patienten-Serum mit Doppelbestimmung innerhalb von 24 Stdn., +++ Enza-trol (Dade)

\begin{tabular}{|c|c|c|c|c|c|c|c|}
\hline \multirow{3}{*}{$\begin{array}{l}\text { Autoren } \\
\text { Kontrollserum }\end{array}$} & \multirow{2}{*}{\multicolumn{2}{|c|}{$\begin{array}{c}\text { Sckily u. Mitarbeiter (17) } \\
\text { Tübingen } \\
\text { Pool-Serum }\end{array}$}} & \multirow{3}{*}{$\begin{array}{c}\underset{\text { Biel }}{\text { BüTTER (1) }} \\
\text { V\% }\end{array}$} & \multirow{2}{*}{\multicolumn{2}{|c|}{$\begin{array}{l}\text { KREUTZ u. ENGLHARDT } \\
\text { Marburg (unveröff.) } \\
\text {-trol (Dade) }\end{array}$}} & \multirow{2}{*}{\multicolumn{2}{|c|}{$\begin{array}{c}\text { ENGLHARDT Düsseldorf } \\
\text { (unveröff.) } \\
\text { Moni-trol (Dade) }\end{array}$}} \\
\hline & & & & & & & \\
\hline & n & V\% & & $n$ & $\mathrm{~V} \%$ & $\mathbf{n}$ & v\% \\
\hline $\begin{array}{l}\text { Harnstoff } \\
\text { Bilirubin } \\
\text { Glucose } \\
\text { Phosphor } \\
\text { Harnsäure } \\
\text { Kalium } \\
\text { Natrium } \\
\text { Chlorid }\end{array}$ & $\begin{array}{l}\frac{10}{10} \\
\frac{10}{-}\end{array}$ & $\begin{array}{l}3,8 \\
\overline{ } \\
4,3 \\
12,4 \\
=\end{array}$ & $\begin{array}{l}3,0 \\
3,7 \\
2,5 \\
\overline{2} \\
2,0 \\
1,4 \\
0,9\end{array}$ & $\begin{array}{r}\frac{160}{160} \\
160 \\
\frac{90}{138} \\
52\end{array}$ & $\begin{array}{l}2,89 \\
3,30 \\
3,87 \\
1,07 \\
0,67 \\
1,19\end{array}$ & $\begin{array}{l}60 \\
60 \\
60 \\
60 \\
60 \\
60 \\
60 \\
60\end{array}$ & $\begin{array}{l}2,8 \\
2,5 \\
3,5 \\
3,9 \\
3,9 \\
3,3 \\
2,2 \\
0,9\end{array}$ \\
\hline $\begin{array}{l}\text { Alanintransaminase } \\
\text { Aspartattransaminase } \\
\text { Lactatdehydrogenase } \\
\text { Alkalische Phosphatase }\end{array}$ & $\frac{10}{12}$ & $\frac{3,8^{+}}{4,5}$ & $\begin{array}{r}8,0 \\
6,0 \\
10,0 \\
7,0\end{array}$ & $\begin{array}{l}162 \\
\frac{172}{165}\end{array}$ & $\begin{array}{l}5,4^{++} \\
\frac{5,7}{3,4}\end{array}$ & $\begin{array}{l}60 \\
60 \\
60 \\
60\end{array}$ & $\begin{array}{l}8,44^{+++} \\
7,97 \\
8,76 \\
11,09\end{array}$ \\
\hline
\end{tabular}


Tab. 6 Qualitätskontrolle von Enzymen bei Verwendung verschiedener $\begin{gathered}\text { Kontroll-Seren aus dem Laboratorium der Medizinischen Universitätsklinik } \\ \text { Marburg }\end{gathered}$

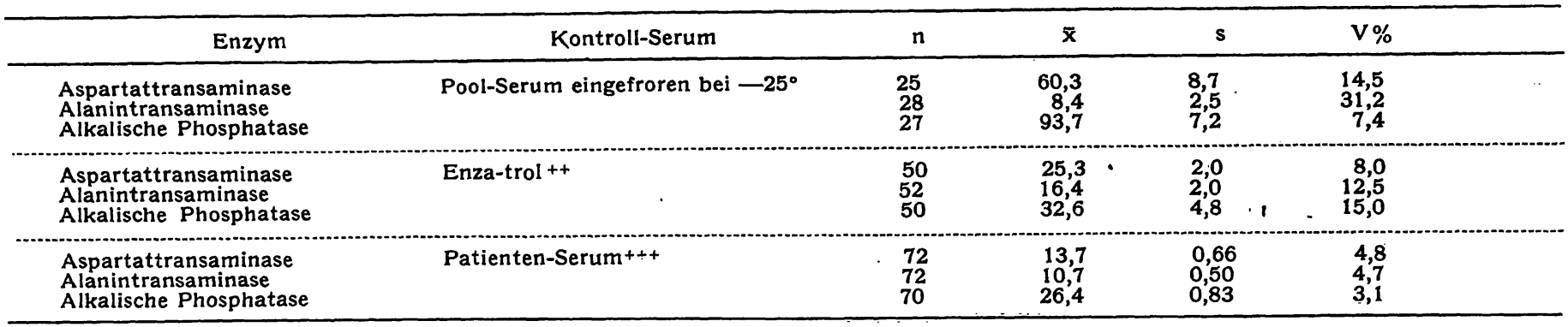

+ Das Zahlenmaterial wurde von Herrn Dr. KREUTz dankenswerterweise zur Verfügung gestellt.

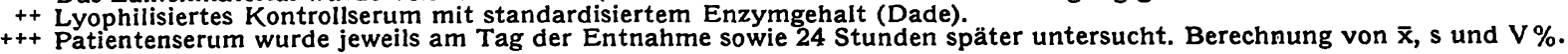

\section{Diskussion}

In vorliegenden Untersuchungen wurden die bei Enzymmessungen im Verlauf der Analyse entstehenden Fehlerquellen geprüft. Ein Teil dieser Fehler ist vermeidbar, z. B. falsche oder variable Meßtemperaturen, Messung bei ungenügendem Vorlauf. Ihr Auftreten zeigt, daß gegen wesentliche Prinzipien der Enzymmessung verstoßen wurde. Ähnlich sind weitere Fehler zu werten, z. B. nicht vorschriftsmäßige Reinigung der Glaswaren. Ein weiterer Teil der Fehler kann aber durch Verbesserung der apparativen Ausrüstung des Laboratoriums reduziert werden. Der Vorteil einer automatischen Registrierung für die Präzision der Messung konnte statistisch gesichert werden. $\mathrm{Ob}$ die Beschaffung eines solchen Geräts rentabel ist, hängt allerdings von der Analysenzahl des Laboratoriums ab. Laboratorien mit einfacher apparativer Ausrüstung sollten daher die Grenzen ihrer Möglichkeiten in der Enzymdiagnostik kennen. Nicht sicher zu beurteilen sind dort vor allem gering erhöhte Werte, bei denen ein ungünstiges Verhältnis zwischen der Größe des methodischen Fehlers und der Variationsbreite des Normbereichs besteht.

Es gibt eine weitere Reihe von Fehlern, die zwar vermeidbar sind, aber im Interesse einer raschen Durchführung großer Meßserien in der Regel in Kauf genommen werden. Zu diesen gehören Fehler, 'die bei der Auswertung auftreten. Dieser Vorgang der Auswertung ist für Enzymmessungen spezifisch. Bei anderen Laboruntersuchungen, soweit sie mit manueller Technik durchgeführt werden, spielt er keine Rolle. Da aber, wie gezeigt wurde, der Auswertfehler bei Enzymmessungen einen hohen Anteil am Gesamtfehler zeigt, ist dieser bei Enzymmessungen schon aus diesem Grund höher als bei anderen klinisch-chemischen Methoden. Dazu kommen auch bei optimaler Technik noch die unvermeidbaren Fehler der Analyse selbst, die denen anderer Methoden entsprechen und die Streuung von Tag zu Tag beeinflussen. Der Anteil dieser Fehler kann reduziert werden, wenn die einzelnen mit Fehlern behafteten Schritte, besonders die Pipettierungen, reduziert werden.

Es muß diskutiert werden, daß die Variationskoeffizienten von Tag zu Tag durch den Vorgang des Lösens des Kontrollserums oder des Auftauens der eingefrorenen Probe beeinflußt werden. Da Enzyme hiergegen möglicherweise empfindlicher sind als andere in diesen Proben enthaltene Substanzen, entsteht hier ein nur Kontrollseren betreffender spezifischer Febler.

Die Präzision einer Methode ist kein für alle Konzentrationsbereiche gültiger Wert. BürtNeR (16) hat bereits auf die Konzentrationsabhängigkeit der Präzision im allgemeinen hingewiesen. Nach FrEI ist diese bei Enzymmessungen besonders ausgeprägt (12). Abbildung 4 zeigt das Ansteigen des Variaționskoeffizienten

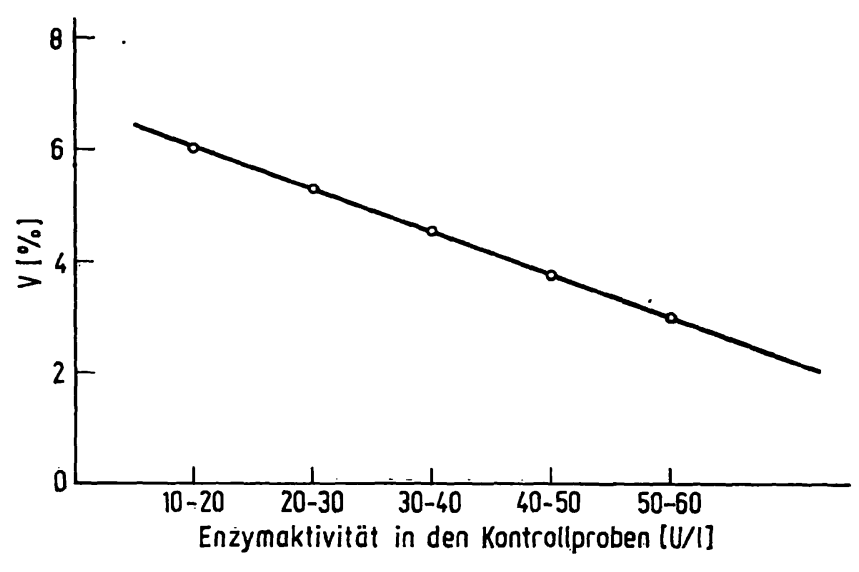

Abb. 4

Konzentrationsabhängigkeit der Präzision von Enzymmessungen (Aspartattransaminase). Variationskoeffizienten bei Doppelanalysen mit zeitlichem Intervail aus Patientënseren verschiedener Aktivität

gegen den Normbereich bei der Messung der Aspattattransaminase. Aus diesem Grund sollen für Kontrolluntersuchungen mit Patientenseren (Doppelbestimmung an zwei hintereinanderfolgenden Tagen) Seren mit mäßig erhöhtem Enzymgehalt verwendet werden. Seren mit sehr hohen Aktivitäten zeigen häufig eine verminderte Stabilität, die vielleicht durch einen hohen Gehalt an Proteasen erklärt werden kann.

Wenn alle vermeidbaren Fehler vermieden werden, kann die Präzision der Enzymmessungen auch über längere Zeit in einem vertretbaren Bereich gehalten werden. Abbildung 5 zeigt eine längere Kontrollperiode aus dem Laboratorium der Universitätsklinik Marburg. Während dieser Kontrolle über 12 Monate 


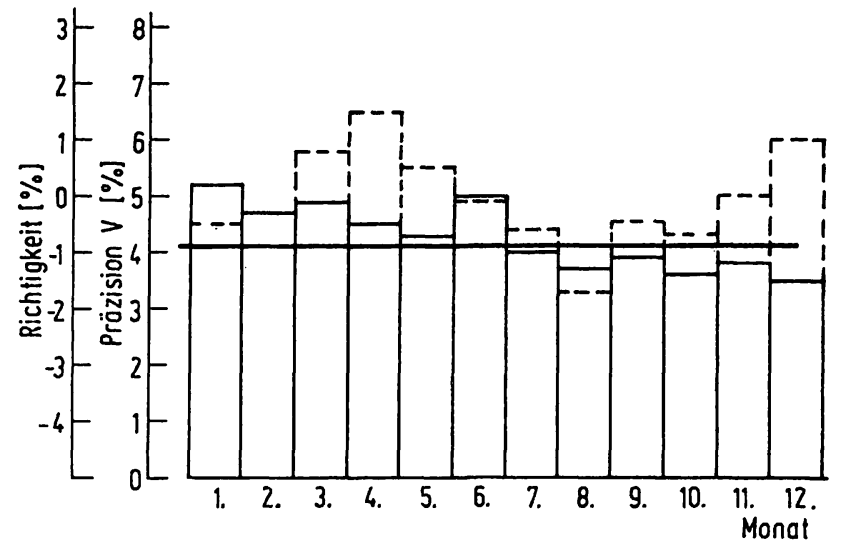

Abb. 5

Präzision ( $\rightarrow$ ) und Richtigkeit $(--)$ bei langfristiger Kontrolle von Enzymmessungen (Aspartattransaminase). UV-Test, Küvetten(Atron. Kontrolle mit Patientenseren mit der Methode der Doppelbestimmungen mit zeitDie ausgezogene Linie gibt die Anforderung des CAP (College of
American Pathologists an

war der Variationskoeffizient nur einmal höher als $6 \%$.

Die von viclen Untersuchern beobachteten hohen Variationskoeffizienten haben wahrscheinlich mehrere
Ursachen, die sicher nicht in allen Laboratorien dieselben sind, da sie von der Methodik und apparativen Ausrüstung des Laboratoriums abhängig sind (z. B. Testansatz, Einsatz zuverlässiger Thermostaten und Kompensationsschreiber, Verwendung von Poolseren oder Kontrollseren unterschiedlicher Stabilität usw.). Es muß damit gerechnet werden, daß die Präzision im Normbereich noch niedriger liegt wegen der bekannten Abhängigkeit der Präzision photometrischer Ablesungen von der Absorption (14). Dies ist eine für alle Präzisionskontrollen gültige Regel (15). Dies ist von besonderer Bedeutung für die beiden Transaminasen, deren Normbereich besonders niedrig liegt. Wenn man die von FreI im Normbereich angegebenen Variationskoeffizienten für die Qualitätskontrolle von Enzymen zugrunde legt (z. B. 19,6\% für die Alanintransaminase), so würden die methodischen Fehler im Norm- und Grenzbereich so hoch sein, daß die Zuverlässigkeit der Methode zur Erfassung gering pathologischer Werte in Frage gestellt wäre. Die Ergebnisse von KREUTZ (18) und eigene Untersuchungen (Tab. 6 u. Abb. 3) haben aber gezeigt, daß bei Verwendung frischer Seren auch im Normbereich wesentlich bessere Variationskoeffizienten erreicht werden können.

\section{Literatur}

1. Bürtner, H., diese Z. 5, 41 (1965). - 2. Kolb, H., Ch. Meyer, R. Merten und H. G. SolbaCh, Mitteilungsdienst GBK 2, 375 (1961). - 3. Löhr, G. W., H. Waller und R. Gross, Dtsch. Med. Wschr. 86, 897 (1961). - 4. SolbaCh, H. G., A. ENGLHARDT und R. Merten, Mitteilungsdienst GBK 2, 375 (1951). - 5 . Feissli, S., G. Forster, G. Laudahn, E. Schmidt und F. W. Schmidt, Klin. Wschr. 44, 390a (1966). - 6. Rertman, S. und B. FränKel, Amer. J. Clin. Path. 28, 56 (1957). - 7. Karmen, A., F. Wroblewski und J. LAdue, J. Clin. Invest. 34, 126 (1955). 8. Wroblewski, F. und J. LAdUe, Proc. Soc. exp. Biol. Med. 90, 210 (1955). - 9. RosalskI, S. B. und J. H. WILKINSON, Nature London 188, 1110 (1960), zit. nach: Richterich, Klinische Chemie, Akadem. Verlagsgesellschaft, Frankfurt/Main (1965). - 10.
Bessey, O. A., O. H. Lowry und M. J. Brock, J. biol. Chemistry 164, 321 (1946), zit. nach: Richterich, Klinische Chemie, Akademische Verlagsgesellschaft, Ffm. (1965). - 11. HARkNEss, J., B. W. Roper, J. A. Durant und H. Miller, Brit. Med. J. 1787 (1960). - 12. Doerffel, K., Beurteilung von Analysenverfahren Springer-Verlag, Berlin (1962). - 13. FreI, J., Z. analyt. Chem. 243, 751 a (1968). - 14. Twrlan, F. und G. F. Lothian, Proc. Physiol. 45, 643 (1933). - 15. Gowentock, A. H. und P. M. G. Broughton, Z. analyt. Chem. 243, 774 (1968). - 16. Bürtner, H., diese Z. 7, 89 (1968). - 17. Schily K., M. EgGstein, W. Knodel, R. Allner, S. Henig und E. Kuhlmand, dise Z. 7, 66 (1969). - 18. KREUTZ, F., unveröffentlicht.
Prof. Dr. Anneliese Englhardt 355 Marburg/Lahn Emil-Mannkopff-Str. 1 